\title{
Exact ${ }^{4}$ He Spectral Function in a Semirealistic NN Potential Model
}

\author{
Victor D. Efros ${ }^{1)}$, Winfried Leidemann ${ }^{2,3)}$, and Giuseppina Orlandini ${ }^{2,3)}$ \\ 1) Russian Research Centre "Kurchatov Institute", Kurchatov Square 1, 123182 Moscow, Russia \\ 2) Dipartimento di Fisica, Università di Trento, I-38050 Povo (Trento), Italy \\ 3) Istituto Nazionale di Fisica Nucleare, Gruppo collegato di Trento, Italy
}

(October 2, 2018)

\begin{abstract}
The spectral function of ${ }^{4} \mathrm{He}$ is calculated with the Lorentz integral transform method in a large energy and momentum range. The excitation spectrum of the residual $3 \mathrm{~N}$-system is fully taken into account. The obtained spectral function is used to calculate the quasi elastic longitudinal $\left(e, e^{\prime}\right)$ response $R_{L}$ of ${ }^{4} \mathrm{He}$ for $q=300,400$, and $500 \mathrm{MeV} / \mathrm{c}$. Comparison with the exact $R_{L}$ shows a rather sizeable disagreement except in the quasi elastic peak, where the differences reduce to about $10 \%$ at $q=500 \mathrm{MeV} / \mathrm{c}$. It is shown as well that very simple momentum distribution approximations for the spectral function provide practically the same results for $R_{L}$ as the exact spectral function.
\end{abstract}

PACS numbers: 25.30.Fj, 21.45.+v, 21.10.Jx, 27.10.+h 
Data on electromagnetic processes on nuclei can be analyzed in a very simple way with the help of a spectral function (SF). The approximations involved in such an analysis are few and transparent. There exists an extensive literature dealing with evaluations and applications of the SF to exclusive, semiinclusive or inclusive reactions [1]. However, only for 3-body nuclei exact calculations of the SF have been performed [2]. A complete evaluation is very difficult for $A>3$ since it requires the knowledge of the complete set of eigenstates for the (A-1)-subsystem. In fact only the (A-1) ground state is often known accurately, while excited states, especially those belonging to the continuum, are much less under control, if not completely unknown. Already for ${ }^{4} \mathrm{He}$ one finds only approximate evaluations of the SF [3], where the final state interaction in the residual 3N-system is neglected. So the quality of the approximations which make use of the SF is often obscured by its poor knowledge.

Applying the method of the Lorentz integral transform [4] one can reduce the complexity of the calculation of the SF considerably. In the present work we use this method to calculate the full SF of ${ }^{4} \mathrm{He}$ with the semirealistic Trento (TN) potential model (central force describing ${ }^{1} S_{0}$ and ${ }^{3} S_{1}$ phase shifts up to pion threshold). The result obtained is then used to evaluate the PWIA longitudinal $\left(e, e^{\prime}\right)$ response function $R_{L}$ at intermediate momenta. The resulting $R_{L}$ 's are compared with the exact ones from Ref. [5] for the same NN potential. Such a comparison enables us to draw conclusions about the precision of the SF ansatz in inclusive $\left(e, e^{\prime}\right)$ scattering within a non relativistic framework. Since ${ }^{4} \mathrm{He}$ is the lightest tightly bound nucleus, these results may be significant also for more complex nuclei.

The spectral function $S(k, E)$ represents the joint probability of finding a particle with momentum $\mathbf{k}$ and a residual (A-1)-system with energy $E$. The momentum $\mathbf{k}$ and the energy $E$ are taken with respect to the c.m. and the ground state of the A-system, respectively:

$$
S(k, E)=\frac{1}{2 J_{0}+1} \sum_{f, s_{z}, t_{z}, M_{0}}\left|\left\langle\psi_{f}^{A-1} ; \mathbf{k} s_{z} t_{z} \mid \psi_{0}^{A}\left(J_{0} M_{0}\right)\right\rangle\right|^{2} \delta\left(E-\left(E_{f}^{A-1}-E_{0}^{A}\right)\right) .
$$

Here $s_{z}$ and $t_{z}$ are the third components of the particle spin and isospin; $E_{f}^{A-1}$ and $\psi_{f}^{A-1}$ are eigenvalues and eigenstates of the (A-1)-system; $J_{0}, M_{0}$ and $E_{0}^{A}$ are the total angular momentum, its third component and the ground state energy of the A-system, respectively. There is a certain number of sum rules the SF has to fulfill:

$$
\begin{gathered}
\int d \mathbf{k} d E S(k, E)=\int d \mathbf{k} n(k)=1, \\
\frac{1}{2 m} \int d E d \mathbf{k} \mathbf{k}^{2} S(k, E)=\langle T\rangle,\langle E\rangle=\frac{A-2}{A-1}\langle T\rangle-2 \frac{E_{0}^{A}}{A} .
\end{gathered}
$$

Here $n(k)$ is the momentum distribution of the A-particle system and $\langle T\rangle$ is the mean kinetic energy of a particle in the ground state. The last relation in Eq. (3) is the so called Koltun sum rule for the mean separation energy [6]. These sum rules form a set of constraints to test the accuracy of a calculation of $S(k, E)$.

In the following we will consider the proton spectral function $S_{p}(k, E)$. In this case the first two sum rules of Eqs. $(2,3)$ have to be modified by an additional factor $Z / A$ on the right-hand sides.

In order to express the one-body knock out cross section in terms of the SF two approximation are required: (i) the particle interacting with the external probe is the one 
detected in experiment, (ii) this particle does not interact with the residual (A-1)-system (PWIA). With these two assumptions the exclusive or semiinclusive one-body knock out cross sections can be written in a factorized form $\sigma \simeq C A \sigma_{N} S\left(\left|\mathbf{k}_{\mathbf{f}}-\mathbf{q}\right|, E\right)$. Here $\sigma_{N}$ is the elementary cross section of the knocked out particle, $\mathbf{k}_{\mathbf{f}}$ is its momentum in the lab-system, $\mathbf{q}$ is the momentum transfer, $E$ is the missing energy, and $C$ is a kinematical factor. The so called quasi elastic (q.e.) cross section can also be written in terms of $S(k, E)$ under the above assumptions. In particular the longitudinal response entering the $\left(e, e^{\prime}\right)$ cross section reads

$$
R_{L}(q, \omega) \simeq A \tilde{G}_{p}^{2}\left(q_{\mu}^{2}\right) \int d \mathbf{k}_{\mathbf{f}} d E S_{p}\left(\left|\mathbf{k}_{\mathbf{f}}-\mathbf{q}\right|, E\right) \delta\left(\omega-E-\frac{\mathbf{k}_{\mathbf{f}}^{2}}{2 m}-\frac{\mathbf{k}_{A-1}^{2}}{2 M_{A-1}}\right),
$$

where $\omega$ is the energy transfer, $q_{\mu}^{2}=q^{2}-\omega^{2}, \tilde{G}_{p}$ is the free proton electric form factor [7], while $M_{A-1}$ and $\mathbf{k}_{A-1}=\mathbf{q}-\mathbf{k}_{\mathbf{f}}$ are mass and recoil momentum of the (A-1)-system, respectively. Here we do not consider an off-shell nucleon form factor, since our aim is a consistent comparison to the full $R_{L}$ of Ref. [5], where such effects were not considered. The definition above includes only the proton responses of the nucleus. In principle one has also to consider the neutron responses, but at low and intermediate $q$ the neutron electric form factor is negligible $\left(\left(\tilde{G}_{n} / \tilde{G}_{p}\right)^{2} \simeq 1 \%\right.$ at $\left.q_{\mu}^{2}=(500 \mathrm{MeV} / \mathrm{c})^{2}\right)$.

The SF can be calculated with the Lorentz integral transform method [4] as already pointed out in Ref. [8]. Let us first denote the overlap of the $A$-body bound state with the single nucleon plane wave

$$
\chi_{p / n}\left(\mathbf{k} ; s_{z}, M_{0}\right)=\left\langle\mathbf{k}, s_{z}, t_{z}= \pm 1 / 2 \mid \psi_{0}^{A}\left(J_{0} M_{0}\right)\right\rangle .
$$

It represents a localized state in the subspace pertaining to the residual (A-1)-subsystem. Written in terms of this quantity, the proton SF

$$
S_{p}(k, E)=\frac{1}{2 J_{0}+1} \sum_{f, s_{z}, M_{0}}\left|\left\langle\psi_{f}^{A-1} \mid \chi_{p}\left(\mathbf{k} ; s_{z}, M_{0}\right)\right\rangle\right|^{2} \delta\left(E-\left(E_{f}^{A-1}-E_{0}^{A}\right)\right),
$$

looks similar to a response function of the (A-1)-subsystem with $\hat{O} \psi_{0}^{A-1}$ replaced by $\chi_{p}$. Therefore we can proceed by analogy with the calculation of a response function. We obtain $S_{P}(k, E)$ as a solution to the integral equation

$$
\int \frac{S_{p}(k, E)}{\left(E-\sigma_{R}\right)^{2}+\sigma_{I}^{2}} d E=\Phi_{p}\left(k, \sigma_{R}, \sigma_{I}\right)
$$

whose right-hand side is given by

$$
\Phi_{p}\left(k, \sigma_{R}, \sigma_{I}\right)=\frac{1}{2 J_{0}+1} \sum_{s_{z}, M_{0}}\left\langle\tilde{\Psi}_{p}\left(\mathbf{k} ; s_{z}, M_{0}\right) \mid \tilde{\Psi}_{p}\left(\mathbf{k} ; s_{z}, M_{0}\right)\right\rangle,
$$

where $\tilde{\Psi}_{p}$ is a localized solution to the 3 -body inhomogeneous equation

$$
\left(H_{A-1}-E_{0}^{A}-\sigma_{R}+i \sigma_{I}\right) \tilde{\Psi}_{p}\left(\mathbf{k} ; s_{z}, M_{0}\right)=\chi_{p}\left(\mathbf{k} ; s_{z}, M_{0}\right) .
$$

We solve Eq. (9) expanding $\tilde{\Psi}_{p}$ in hyperspherical harmonics. Complete convergence of the expansion is reached with similar values for the expansion parameters as in Ref. [5]. The 
inversion of the Lorentz integral transform, Eq. (7), is carried out as described in Ref. 《4. Quite a good stability of the inversion results is observed. As previously we check the quality of the results with the help of sum rules as well. To this end we evaluate the sum rules of Eqs. $(2,3)$ by an explicit integration of the properly weighted calculated SF. We obtain the following relative differences $0.9 \%$ (norm), $0.2 \%(\langle T\rangle$ ), and $0.8 \%$ (Koltun sum rule assuming that $\left.S_{n}(k, E)=S_{p}(k, E)\right)$. Since the sum rules weight $S(k, E)$ in different regions these results point out that the $\mathrm{SF}$ is calculated with a satisfying precision.

Before coming to the SF, in Fig. 1 we show the $n(k)$ of ${ }^{4} \mathrm{He}$ for the TN potential in comparison to that obtained for a realistic potential (Argonne $v_{18}+$ Urbana IX) [9]. One sees a rather good agreement up to almost $2 \mathrm{fm}^{-1}$. However, different from the realistic result the semirealistic $n(k)$ is considerably smaller at higher $\mathrm{k}$. Most of these differences are presumably explained by the missing tensor force in the TN potential (see Ref. [10]). We also show in Fig. 1 two partial momentum distributions. They are obtained from Eqs. $(1,2)$ if the sum over $f$ in Eq. (1) is restricted either to the triton ground $\left(n_{t p}(k)\right)$ or its continuum state $\left(n_{t^{*} p}(k)\right)$. As expected (see Ref. [1]) $n_{t p}(k)$ governs the lower $k$ momentum distribution, while $n_{t^{*} p}(k)$ dominates at higher $k$. The integration of $n_{t p}(k)$ leads to the so called spectral factor. For the TN potential one finds a spectral factor of 0.89 , whereas with the above realistic potential a value of 0.84 [9] is obtained.

In Fig. 2 we show $S_{p}(k, E)$. Only energies above the breakup threshold $E_{t h r}^{A-1}$ of the rest nucleus are illustrated, while the contribution from the bound state of the rest nucleus is identical to the $n_{t p}(k)$ of Fig. 1 (see Eq. (1)). The values of $S\left(k, E \geq E_{t h r}^{A-1}+1 \mathrm{MeV}\right.$ ) are plotted in the figure. We note that $S\left(k, E_{t h r}^{A-1}\right)=0$, and thus $S(k, E)$ exhibits a rather strong slope at low energy. For momenta below $2 \mathrm{fm}^{-1}$ one finds a sharp maximum at about $2 \mathrm{MeV}$ above $E_{t h r}^{A-1}$. On the contrary $S(k, E)$ is flat in most other regions. Only for $k>2$ $\mathrm{fm}^{-1}$ there is a ridge where the peak position shifts to higher $E$ for increasing $k$.

As already mentioned one of our aims is a comparison of the q.e. $R_{L}$ with the exact one in an intermediate $q$ range for the same NN potential. In Fig. 3 we show both $R_{L}$ 's in comparison to experimental data. We would like to point out that the full results are a bit different from those in Ref. [5] for two reasons: (i) in the calculations of Ref. [5] $G_{n}\left(q_{\mu}^{2}\right)$ entered erroneously with a negative sign leading to small - but not totally negligible - effects on $R_{L}$ (e.g., peak height and high-energy tail become a bit lower); (ii) different from Ref. [5] here we account for the small overbinding of the TN potential for ${ }^{4} \mathrm{He}$. The threshold energy reads $\omega_{t h r}=E_{0}\left({ }^{3} \mathrm{H}\right)-E_{0}\left({ }^{4} \mathrm{He}\right)+q^{2} / 2 M\left({ }^{4} \mathrm{He}\right)$ and we correct the overbinding by shifting our response to lower energies to make $\omega_{t h r}$ correspond to the one with the experimental $E_{0}\left({ }^{4} \mathrm{He}\right)$.

Of course these two modifications do not change the general picture given in Ref. [5]. The good agreement with experiment becomes even better for q.e. peak and high-energy tail. At low energy there is a slight improvement at $q=300 \mathrm{MeV} / \mathrm{c}$. For the two higher $q$ the agreement with experiment in the threshold region is still satisfying but not as excellent as shown in Ref. [5].

Figure 3 shows, as expected, that at $q=300 \mathrm{MeV} / \mathrm{c}$ the PWIA does not lead to a good description of $R_{L}$. The q.e. peak is shifted to higher energies by $15 \mathrm{MeV}$ and the peak height is overestimated by more than $40 \%$. The overestimation becomes worse with increasing energy, while at low energy $R_{L}$ is underestimated. At the two higher $q$ the peak is shifted by $12 \mathrm{MeV}$, but since the peak width grows with increasing $q$ this shift is a minor 
effect. The shift of the peak can be qualitatively understood considering a nucleon at rest in a potential well: $\omega$ can be estimated as $q^{2} /(2 m)+V_{f}-V_{i}$, where $V_{i, f}$ are the potential energies before or after interaction with the virtual photon. While $V_{f}$ is negative, it becomes zero in PWIA leading to an increase in $\omega$. The peak height improves with overestimations of $25 \%$ at $q=400$ and $13 \%$ at $500 \mathrm{MeV} / \mathrm{c}$. Thus one has to expect that beyond $500 \mathrm{MeV} / \mathrm{c}$ the PWIA is a good approximation at the q.e. peak. Beyond the peak the PWIA result still overestimates the exact one considerably, but the discrepancy decreases with increasing $q$. At low energy, however, the underestimation remains considerably large.

It is advantegeous to have a simple and good approximation for the PWIA response. From Fig. 2 it is evident that at low $k$ almost all the strength of $S(k, E)$ with the disintegrated rest nucleus is found close to the breakup threshold. This suggests the following approximation

$$
S(k, E) \simeq n_{t p}(k) \delta\left(E-E_{0}\left({ }^{3} \mathrm{H}\right)+E_{0}\left({ }^{4} \mathrm{He}\right)\right)+n_{t^{*} p}(k) \delta\left(E-E_{t^{*} p}+E_{0}\left({ }^{4} \mathrm{He}\right)\right)
$$

for calculating inclusive processes, where $E_{t^{*} p}$ is the breakup energy of the rest nucleus. One obtains an even simpler approximation considering that $E_{t^{*} p} \simeq E_{0}\left({ }^{3} \mathrm{H}\right)$ :

$$
S(k, E) \simeq n(k) \delta\left(E-E_{0}\left({ }^{3} \mathrm{H}\right)+E_{0}\left({ }^{4} \mathrm{He}\right)\right) .
$$

Eq. (11) was used e.g. in Ref. [13] where large deviations from the full GFMC response for a realistic potential at $q=400 \mathrm{MeV} / \mathrm{c}$ were reported. In Fig. 4 we show the PWIA results with the above two approximations at $q=500 \mathrm{MeV} / \mathrm{c}$ relative to the full SF result. It is readily seen that the three responses are very similar, particularly in the q.e. peak region (at $q=300,400$, and $1000 \mathrm{MeV} / \mathrm{c}$ one has very similar results). It is worth mentioning that our PWIA result at $q=400 \mathrm{MeV} / \mathrm{c}$ is essentially the same as the one in Ref. [13]. This shows again that a semirealistic central force leads for $R_{L}$ practically to the same result as a realistic potential.

In this work we obtain for the first time the full spectral function of ${ }^{4} \mathrm{He}$. A semirealistic NN potential is used. The final state interaction in the residual system is taken into account completely by the Lorentz integral transform method. The SF is then used to calculate the q.e. longitudinal response function of ${ }^{4} \mathrm{He}$ which is compared to the exact one of Ref. [5]. In the peak region the differences decrease with growing momentum transfer up to about 10 $\%$ at $q=500 \mathrm{MeV} / \mathrm{c}$, but one still finds sizeable differences apart from the peak. Similar results were found in Ref. [14 for ${ }^{3} \mathrm{H}$ and ${ }^{3} \mathrm{He}$ for the studied momentum transfers of 300 and $400 \mathrm{MeV} / \mathrm{c}$. Different from the 3-body system ${ }^{4} \mathrm{He}$ already resembles some aspects of more complex nuclei and thus the general picture of the q.e. response should not change much in such systems. We show as well that the simple momentum distribution approximations for the SF provide results for $R_{L}$ which are quite close to those obtained with the full SF.

Two of us (W.L. and G.O.) thank the Institute of Nuclear Theory at the University of Washington for its hospitality and the Department of Energy for partial support during the completion of this work. The work of V.D.E. was supported by INFN and RFBR (grants no 96-15-96548 and 97-02-17003). 


\section{REFERENCES}

[1] for a recent review see C. Ciofi degli Atti and S. Simula, Phys. Rev. C 53, 1689 (1996).

[2] A. E. L. Dieperink, T. de Forest, I. Sick, and R. A. Brandenburg, Phys. Lett. B 63, 261 (1976); C. Ciofi degli Atti, E. Pace, and G. Salmé, Phys. Rev. C 21, 805 (1980); H. Meier-Hajduk, P. U. Sauer, and W. Theis, Nucl. Phys. A395, 332 (1983).

[3] H. Morita and T. Suzuki, Prog. Theor. Phys. 86, 671 (1991).

[4] V. D. Efros, W. Leidemann, and G. Orlandini, Phys. Lett. B 338, 130 (1994).

[5] V. D. Efros, W. Leidemann, and G. Orlandini, Phys. Rev. Lett. 78, 432 (1997).

[6] A. E. L. Dieperink and T. De Forest Jr., Ann. Rev. Nucl. Sci., 25, 1 (1975); D. S. Koltun, Phys. Rev. C 9, 484 (1974); S. Boffi, Lett. Nuovo Cimento, 1971 (1971).

[7] T. de Forest Jr., Nucl. Phys. A414 347 (1984).

[8] V. D. Efros, W. Leidemann, and G. Orlandini, Few Body Syst. Suppl. 8, 389 (1995).

[9] R. Schiavilla, V. R. Pandharipande, and R. B. Wiringa, Nucl. Phys. A449, 219 (1986); R.B. Wiringa, Phys. Rev. C 43, 1585 (1991); A. Arriaga, V. R. Pandharipande, and R. B. Wiringa, Phys. Rev. C 52, 2362 (1995); R. B. Wiringa, private communication.

[10] H. Morita, Y. Akaishi, and H. Tanaka, Prog. Theor. Phys. 79, 863 (1988).

[11] S.A. Dytman, A. M. Bernstein, K. I. Blomqvist, T. J. Pavel, B. P. Quinn, R. Altemus, J. S. McCarthy, G. H. Mechtel, T. S. Ueng, and R. R. Whitney, Phys. Rev. C38, 800 (1988).

[12] A. Zghiche et al., Nucl. Phys. A 572, 513 (1994).

[13] J. Carlson and R. Schiavilla, Phys. Rev. Lett. 68, 3682 (1992).

[14] S. Ishikawa, H. Kamada, W. Glöckle, J. Golak, and H. Witala, Phys. Lett. B 339, 293 (1994). 


\section{FIGURES}

FIG. 1. Total (solid curve) and partial momentum distributions $n_{t p}$ (dotted curve) and $n_{t^{*} p}$ (dashed curve) of ${ }^{4} \mathrm{He}$ with TN potential; also shown total result (full dots) and $n_{t p}$ (open squares) with Argonne $v_{18}+$ Urbana IX [9].

FIG. 2. $S_{p}(k, E)$ of ${ }^{4} \mathrm{He}$ with $\mathrm{TN}$ potential in units of $\mathrm{fm}^{3} \mathrm{MeV}^{-1}$.

FIG. 3. $R_{L}$ of ${ }^{4} \mathrm{He}$ with TN potential: PWIA results according to Eq. (4) (dashed curves) and full results (solid curves); experimental data from Bates [11] and Saclay [12].

FIG. 4. $R_{L}$ with SF of Eq. (10) (dashed curve) and of Eq. (11) (dotted curve) relative to $R_{L}$ with full SF (the q.e. peak is marked by an arrow). 


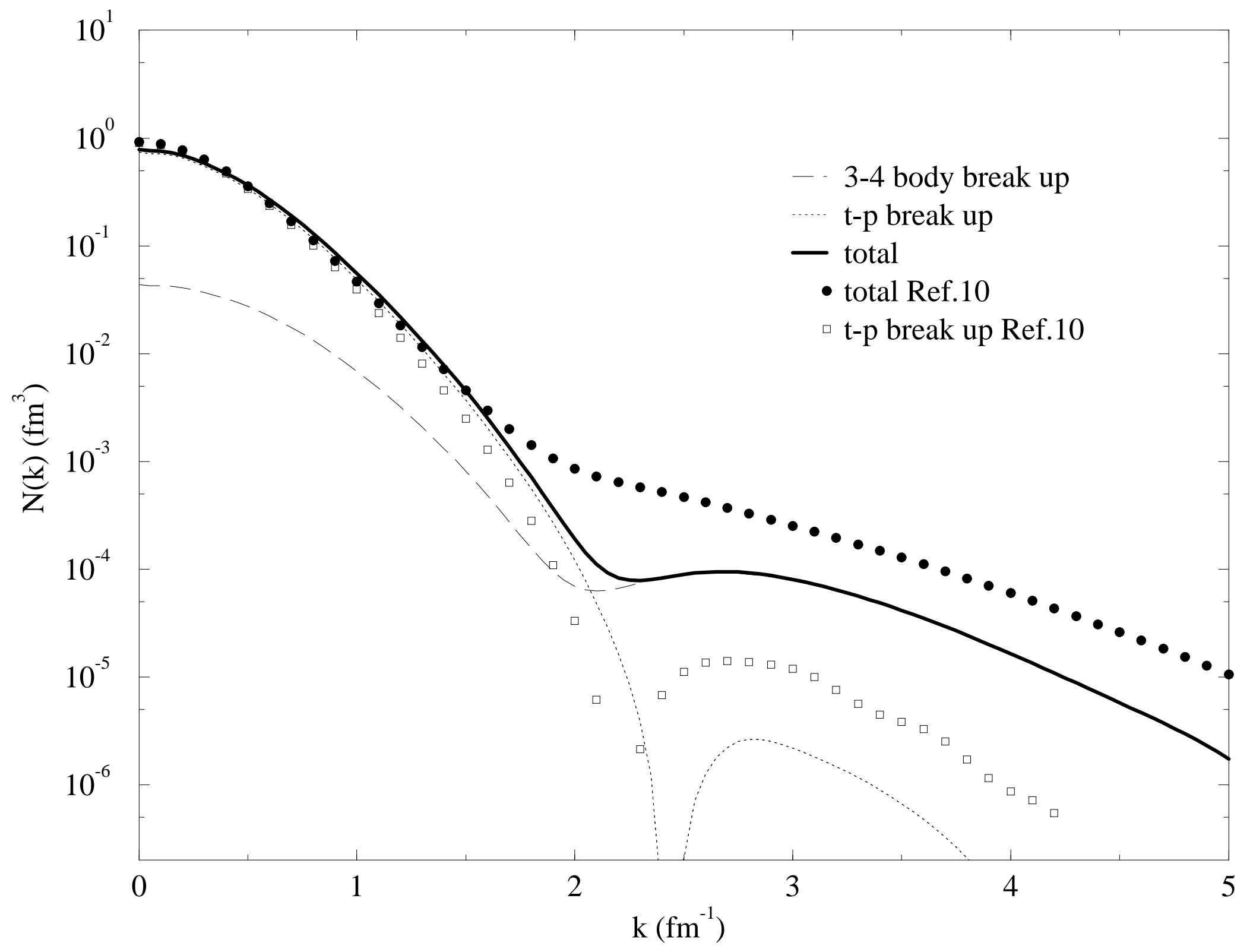




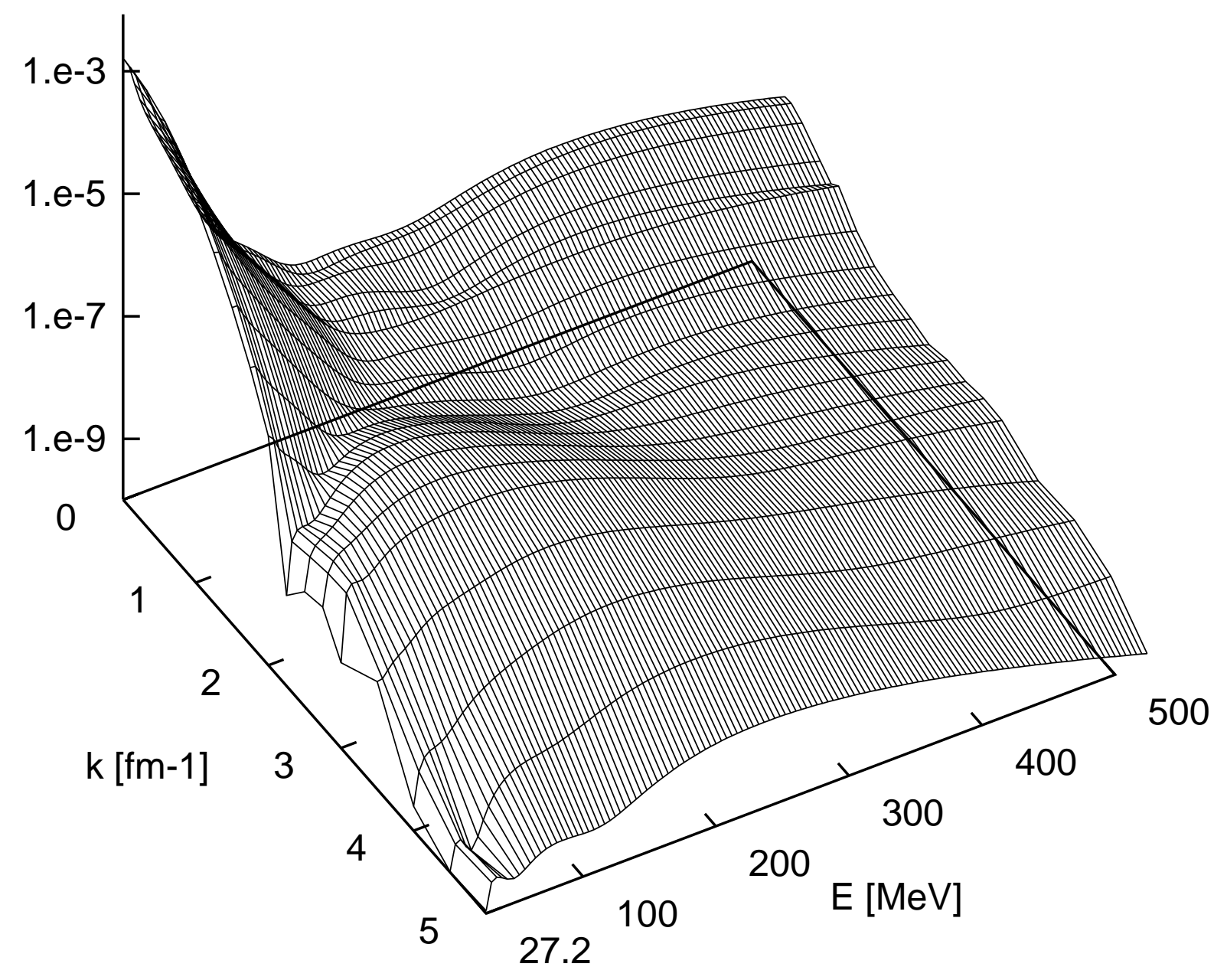



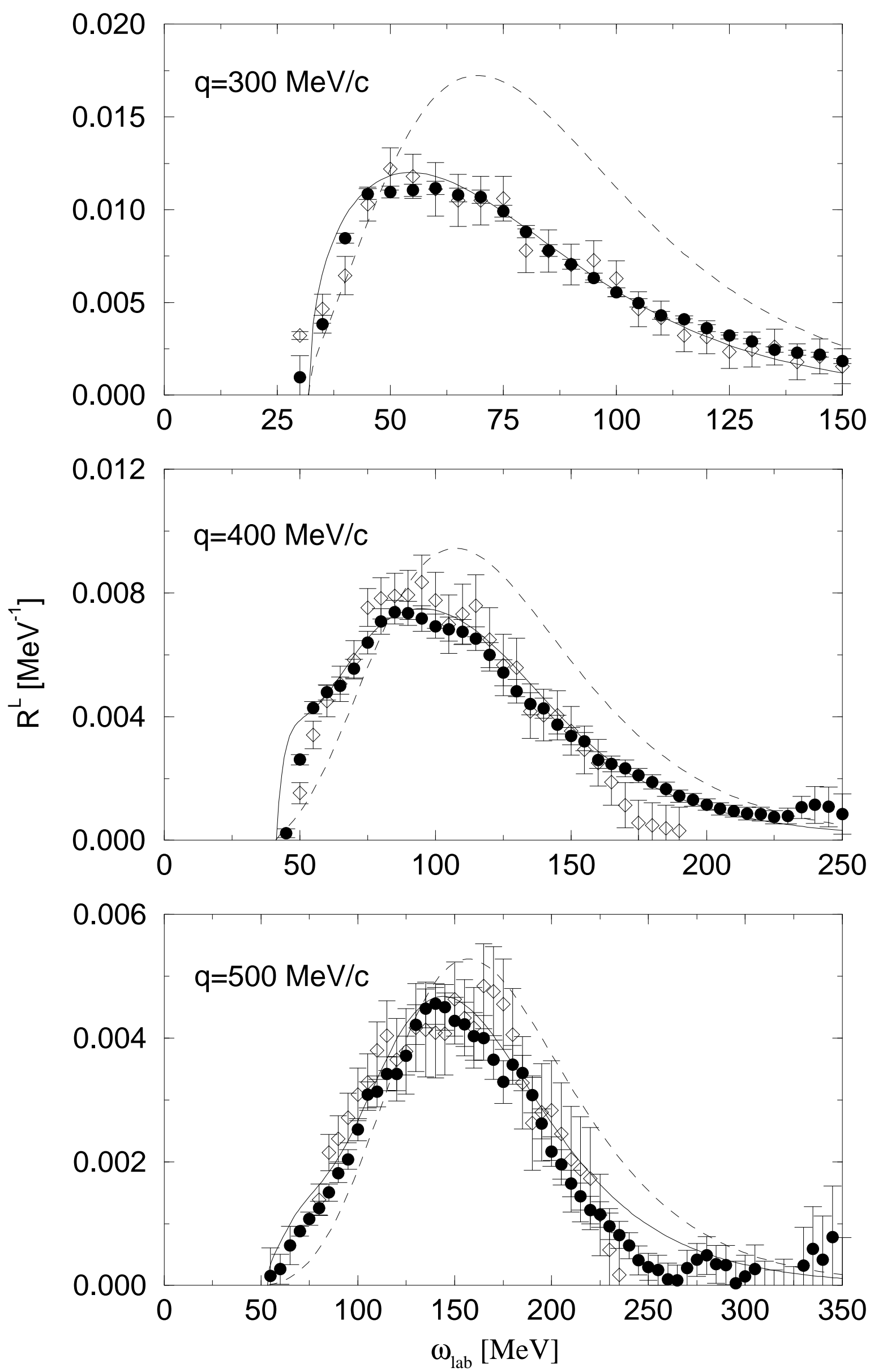


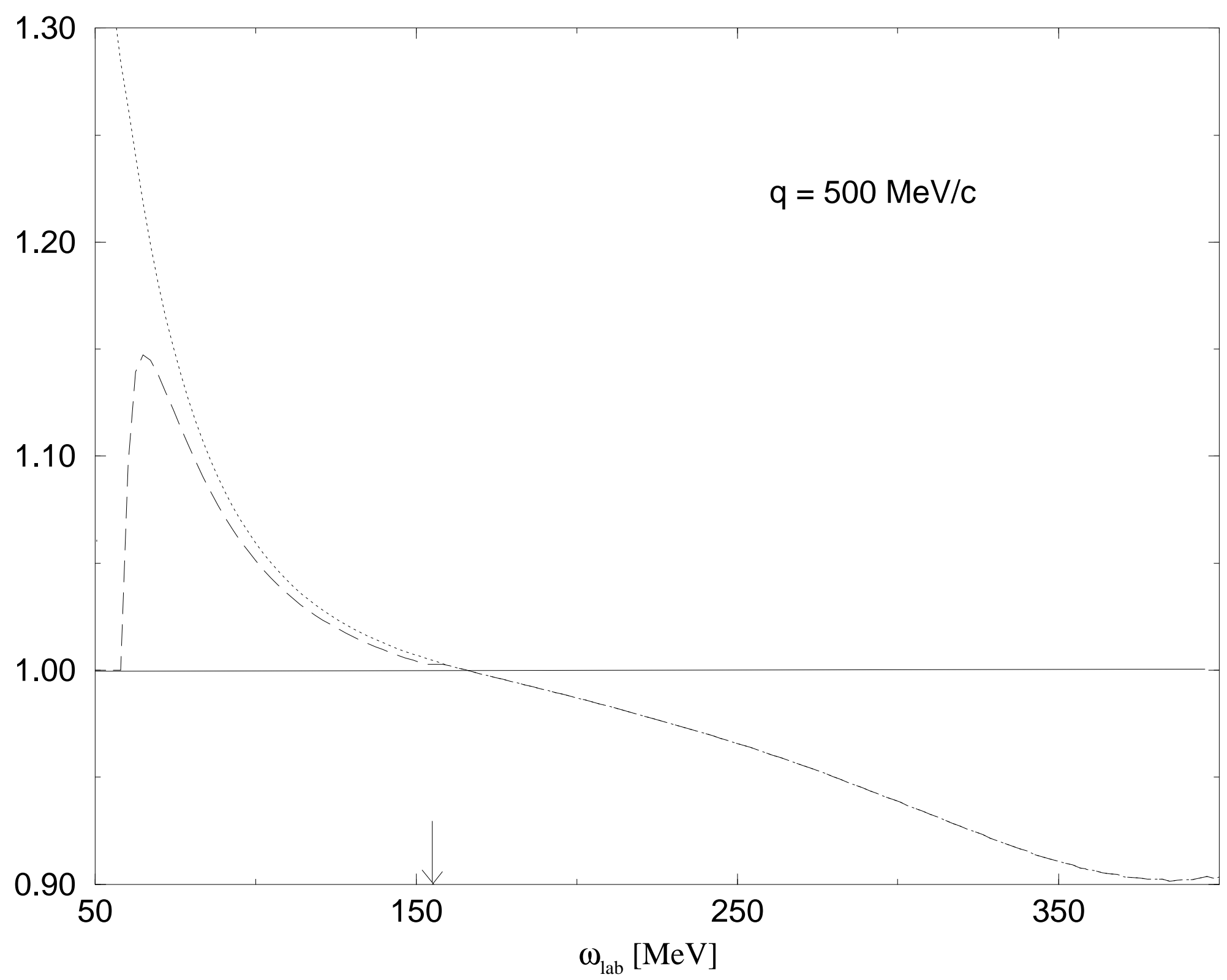

